\title{
EL DESARROLLO RURAL EN LA PROVINCIA DEL CHACO: FUNDAMENTOS Y VARIABLES DE ANÁLISIS.
}

\section{RURAL DEVELOPMENT IN THE PROVINCE OF CHACO: FUNDAMENTALS AND VARIABLES OF ANALYSIS}

\author{
Prof. Mgter. Viviana C. Pertile \\ Profesora Adjunta. Cátedra Geografía Urbana y Agraria \\ Departamento de Geografía. Facultad de Humanidades. Universidad Nacional del Nordeste \\ http://dx.doi.org/10.30972/geo.14282747
}

\begin{abstract}
RESUMEN:
Las políticas públicas de desarrollo rural impulsadas desde hace varias décadas en el territorio provincial tuvieron poca efectividad, ello es evidente, por cuanto los datos recopilados muestran una creciente desigualdad y despoblamiento de amplias zonas rurales de nuestra provincia. En relación a dichas políticas de desarrollo rural, se pudo advertir, que se ha tratado de una serie de propósitos discursivos, por cuanto no fueron diseñadas ni definidas desde los ámbitos locales, prueba de ello son las transformaciones territoriales como así también los contrastes sociales y económicos que se han producido tanto en ámbitos rurales nacionales como provinciales. Nuestro espacio de estudio es el resultado de procesos y de transformaciones históricas que involucran diferentes aspectos tanto demográficos como productivos. Esta situación la podemos explicar mediante el análisis de distintas variables demográficas y socio-económicas que dan cuenta de las necesidades que se deben cubrir en la provincia del Chaco
\end{abstract}

\section{PALABRAS CLAVES}

Desarrollo rural; Chaco;Variables.

\section{ABSTRACT}

The public policies of rural development promoted for several decades in the provincial territory had little effectiveness, that is evident, because the collected data show a growing inequality and depopulation of large rural areas of our province territory. Regarding to these rural development policies, it could be noticed that it has been a series of discursive purposes, because it hasn' t been designed or defined from the local area, proof of that are the territorial transformations as well as the social and economic contrasts. That have occurred in both national and provincial rural areas. Our study space is the result of processes and historical transformations that involve different aspects, both demographic and productive. We can explain that situation through the analysis of different demographic and socio-economic variables that explain the needs that must be serve in the province of Chaco.

\section{KEY WORDS}

Rural development; Chaco; Variables

\section{Introducción}

Desde hace varias décadas se puede advertir que el interior de la provincia del Chaco presenta características socioeconómicas dispares, evidenciadas por una marcada heterogeneidad económica y social. Muchas han sido las causas de esta situación, pero en relación a la temática que en esta oportunidad abordaremos, debemos decir que las 
políticas de desarrollo rural que se vienen implementando en la provincia distan de ser íntegras y duraderas y/o estables. No son íntegras porque los problemas de carácter estructural no han sido resueltos con políticas cuyo alcance abarque la totalidad de los problemas que implican dichas diferencias. Tampoco han sido estables dado que no se implementaron medidas de largo plazo que pudieran revertir la situación de desigualdad.

Por otra parte, la multiplicidad de programas y/o proyectos vigentes determinó un uso ineficaz de los recursos públicos y una gran dependencia de los beneficiarios, llevándolos a la permanente búsqueda de asistencialismo.

Manzanal (2011) sostiene que el Desarrollo rural en la Argentina no fue una política de gobierno, sino que fue una sumatoria de programas que hubo desde 1985 hasta 2007, es decir previo a los conflictos del campo y que es cuando se crea la Secretaría de Desarrollo Rural y Agricultura Familiar y que después se transforma en Ministerio de Agricultura y finalmente a partir del año 2015 se transforma en Secretario de Agr. Familiar, Coordinación y Des. Territorial (1). Lo primero que se plantea en la década del 80 era por qué Argentina tenía que tener planes de desarrollo rural, desde entonces la respuesta tiene que ver con que eran para mejorar la calidad de vida de los pequeños productores y pobres rurales. Esto es alcanzar mejoras en la producción, en la colocación y articulación en los mercados regionales y nacionales, en la organización a través de la promoción y acceso a recursos productivos, crédito, asistencia técnica y capacitación y de esa manera contribuir al alivio de la pobreza en las áreas rurales.

Apoyándonos en Manzanal (op.cit), podríamos decir que los objetivos de una agenda - política rural provincial deberían estar orientados a reducir las diferencias entre las distintas zonas de la provincia e igualar las oportunidades de todos los habitantes del Chaco, sean estos urbanos o rurales.

Por otra parte, la variedad de áreas rurales en nuestro espacio de estudio, es el resultado de procesos de transformaciones históricas que involucran diferentes aspectos como ser los productivos; asimismo debemos considerar que existen diferencias en lo que hace al acceso a la educación en todos sus niveles, a la infraestructura básica, a un sistema de salud eficiente, entre otros, que marcaron las diferencias de oportunidades y el éxito de los territorios.

Esta situación la podemos explicar mediante el análisis de distintas variables demográficas y socio-económicas que dan cuenta de las necesidades que se deben cubrir en la provincia del Chaco y que serán expuestas en párrafos siguientes.

Las periódicas referencias a la situación de abandono de miles de pequeños productores, o a la escasa competitividad que tienen nuestros productos en el mercado interno y externo, tienen al espacio rural como espacio subyacente. En las últimas tres décadas hemos presenciado un creciente proceso de urbanización en la mayoría de las provincias argentinas y particularmente en la provincia del Chaco, siendo las ciudades más importantes testigos de esta situación; la ciudad de Presidencia Roque Sáenz Peña, localizada en el centro-este de la provincia en un claro ejemplo de ello, ya que concentró en los últimos años un cinturón de nuevos barrios y asentamientos (San Cayetano, Mitre, Sarmiento, entre otros) que van conformando un espacio densamente poblado y que además de alimentos, necesita de los servicio públicos como luz, recolección de residuos, agua potable, en muchos casos deficitarios. Es decir que, la conformación de cordones de marginalidad y abandono en las ciudades, constituye otro elemento de este fenómeno. A pesar de ello, la población rural sigue eligiendo como destinos residenciales a éstas ciudades en virtud de que tiene mayor posibilidad de acceder a mayores oportunidades laborales. 
Este período iniciado aproximadamente en los $90^{`}$ del siglo pasado se caracterizó por presentar un débil sector productivo, una población joven y de pequeños productores emigrando de sus predios rurales, con la consecuente declinación de la población rural.

También es preciso reconocer que a lo largo de su historia rural, el Chaco estuvo muy ligado al desarrollo que adquiriesen los centros urbanos en función de las prácticas agrícolas circundantes. Es así que, lo que anteriormente representara el departamento Comandante Fernández (cuya cabecera es Presidencia Roque Sáenz Peña) con su exponente e imponente cultivo de algodón, permitiendo el nacimiento de cooperativas agrícolas como lo fue "la Unión Limitada" fundada en 1937, fue dejando paulatinamente ese lugar ante la debacle económica de principios de los años 1990. Paralelamente comienza a tener apogeo el sudoeste chaqueño, con el departamento Chacabuco y su cabecera Charata, convirtiéndose en un emporio productivo primero con la producción de algodón y luego con la soja. (Pertile, 2004)(2).

Evidentemente los ejes fundamentales para definir las políticas tendientes al desarrollo productivo estarían dados en función de diversificar la actividad económica y ampliar el mercado de trabajo.

En este trabajo se intenta poner de manifiesto algunas variables que se tienen en cuenta al estudiar el desarrollo rural y que están básicamente relacionadas con aquellas que reflejan las características productivas y las condiciones de vida de la población involucrada.

\section{Fundamentos de aplicación de los programas de desarrollo rural}

Lo primero que se plantea en la década del 80 era por qué Argentina tenía que tener planes de desarrollo rural, desde entonces la respuesta tiene que ver con que eran para mejorar la calidad de vida de los pequeños productores y pobres rurales. Esto es alcanzar mejoras en la producción, en la colocación y articulación en los mercados regionales y nacionales, en la organización a través de la promoción y acceso a recursos productivos, crédito, asistencia técnica y capacitación y de esa manera contribuir al alivio de la pobreza en las áreas rurales.

Es así como comienzan a aparecer los Programas, organizados desde distintos organismos:

SAGPyA (Secretaría de Agricultura, Ganadería, Pesca y Alimentación) tuvo a su cargo: PSA (Programa Social Agropecuario), PROINDER (Proyecto de Desarrollo de Pequeños Productores Agropecuarios), PRODERNEA (Programa de Desarrollo Rural de las Provincias del Noreste Argentino), estos tres estuvieron orientados a los pequeños productores, CAPPCA (Proyecto Forestal de Desarrollo), PRODERPA, (Proyecto de Desarrollo Rural de la Patagonia) PRODEAR (Programa de Desarrollo de Áreas Rurales). Ministerio de Economía y Producción (2003).

INTA (Instituto Nacional de Tecnología Agropecuaria) se ocupó de: PROFEDER (Programa Federal de Apoyo al Desarrollo Rural Sustentable, este Programa fue diseñado y opera desde el año 2003 para contribuir a la promoción de la innovación tecnológica y organizacional, el desarrollo de las capacidades de todos los actores del sistema y el fortalecimiento de la competitividad sistémica regional y nacional, en un ámbito de equidad social y sustentabilidad. Su acción se ejecuta mediante proyectos y planes de trabajos grupales en terreno, destinados a satisfacer las demandas y oportunidades que surgen del territorio. La respuesta a la demanda específica se opera mediante distintos instrumentos, con objetivos y metodologías de trabajo propios: Cambio Rural (Programa Federal de Reconversión Productiva para la Pequeña y Mediana Empresa Agropecuaria, si bien se encuentra dentro del PROFEDER, fue creado en el año 1993 por la Secretaría de Agricultura, Ganadería y Pesca), Pro-Huerta (El Pro-Huerta es un programa del INTA y del

Publicado en formato digital: Prof. Mgtr. Viviana Claudia Pertile. EL DESARROLLO RURAL EN LA PROVINCIA DEL CHACO: FUNDAMENTOS Y VARIABLES DE ANÁLISIS. Revista Geográfica Digital. IGUNNE. Facultad de Humanidades. UNNE. Año 14. № 28. Julio - Diciembre 2017. ISSN 1668-5180 Resistencia, Chaco.

En: http://hum.unne.edu.ar/revistas/geoweb/default.htm 
Ministerio de Desarrollo Social de la Nación con más de 20 años de trabajo en territorio, Basado en los principios de la agricultura orgánica,), Proyectos de Apoyo al Desarrollo Local, Minifundio, Profam (Productores familiares, se inicia en el año 2003 y destinados a productores de muy pequeñas escalas, con dificultades para el acceso al crédito y a la comercialización y con bajos ingresos ) y Proyectos Integrados. Todos y cada uno de los instrumentos basan la estrategia en la acción participativa.

\section{Variables de análisis involucradas en el desarrollo rural}

El enfoque territorial del desarrollo rural permitir abordar la problemática rural en la provincia del Chaco, ya que tiene en cuenta un gran número de variables para su análisis y diagnóstico. Si bien la actividad agrícola es central en este estudio, también pueden analizarse otras variables como ser la pobreza rural, analizada mediante el indicador de las Necesidades Básicas Insatisfechas; variables demográficas; variables económicas, entre otras.

Para conocer las características de las condiciones de vida del espacio rural de la provincia del Chaco es necesario analizar un conjunto de variables que permitan indicar si dichas condiciones han cambiado en los últimos años o si las brechas entre lo urbano y lo rural han sido zanjadas o se han profundizado.

En general se conoce que los espacios rurales que lograron posicionarse competitivamente han podido achicar las diferencias en la calidad de vida y oportunidades respecto de las áreas urbanas.

Para este estudio tomaremos como elementos de análisis aquellos que reflejen condiciones de vida y variables económicas.

\section{Población Urbana y Rural de la provincia del Chaco Urbanización}

En el marco del enfoque territorial del desarrollo rural que estamos abordando, donde el territorio está constituido tanto por áreas urbanas como rurales, es común que las mismas sean vistas como dos realidades diferentes. Generalmente se tiende a pensar que el mayor grado de urbanización implica la situación ideal a alcanzar, sin embargo, consideramos que, lo que se tiene que lograr es aplicar políticas que tiendan a mejorar las condiciones de vida de la población residentes en ambos territorios.

Es por ello que, como punto de partida analizamos la distribución y el comportamiento evolutivo de la población en el territorio provincial, a los efectos de detectar otras particularidades como lo son las características socioeconómicas o las variables productivas.

\begin{tabular}{|c|c|c|c|c|}
\hline Años & \multicolumn{2}{|c|}{ Chaco } & \multicolumn{2}{c|}{ Argentina } \\
\hline & $\begin{array}{c}\text { Rural } \\
\%\end{array}$ & Urbana\% & Rural & Urbana\% \\
\hline $\mathbf{1 9 4 7}$ & 69,9 & 30,1 & 37.8 & 62.2 \\
\hline $\mathbf{1 9 6 0}$ & 53,6 & 46,4 & 28.0 & 72.0 \\
\hline $\mathbf{1 9 7 0}$ & 48,8 & $\underline{\mathbf{5 1 , 2}}$ & 21.6 & 78.4 \\
\hline $\mathbf{1 9 8 0}$ & 39,1 & 60,9 & 17.1 & 82.9 \\
\hline $\mathbf{1 9 9 1}$ & 33,4 & 66,6 & 14.7 & 85.3 \\
\hline $\mathbf{2 0 0 1}$ & 20,6 & 179,4 & 10.6 & 89.4 \\
\hline $\mathbf{2 0 1 0}$ & 12,2 & 87,8 & 9.0 & 91.0 \\
\hline
\end{tabular}

El proceso de urbanización del Chaco acompañó, como en gran parte del territorio argentino, las etapas del desarrollo económico y, en las últimas décadas, el fenómeno cultural "ciudad" -tal como la definen numerosos autores- fue el principal motivo de

Publicado en formato digital: Prof. Mgtr. Viviana Claudia Pertile. EL DESARROLLO RURAL EN LA PROVINCIA DEL CHACO: FUNDAMENTOS Y VARIABLES DE ANÁLISIS. Revista Geográfica Digital. IGUNNE. Facultad de Humanidades. UNNE. Año 14. № 28. Julio - Diciembre 2017. ISSN 1668-5180 Resistencia, Chaco. 
atracción de la población a los centros más urbanizados de nuestra provincia. Asimismo debemos decir que este crecimiento en la urbanización respondió fundamentalmente a dos factores, por un lado al crecimiento demográfico propiamente dicho y por otro lado las actividades económicas de la ciudad, las que ofrecen mayores y mejores oportunidades laborales, sumado a ello las deficientes condiciones de vida imperantes en el medio rural del Chaco.

Esto determinó que, como se aprecia en el gráfico № 1, en 2010 el $87,8 \%$ de la población chaqueña vivía en ciudades.

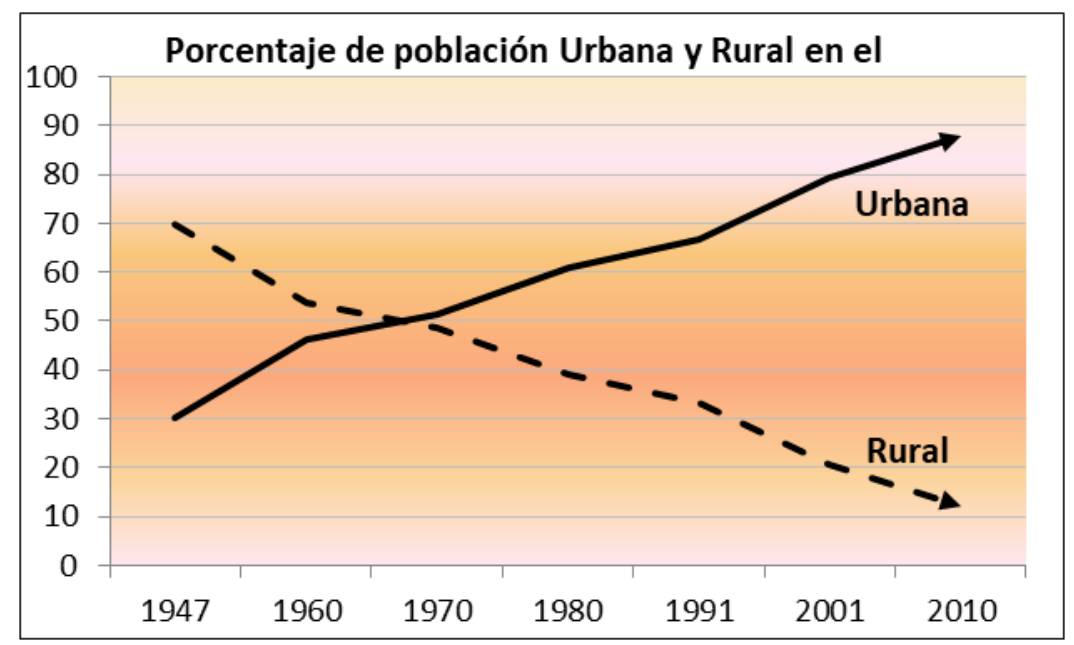

Gráfico № 1
En la provincia del Chaco la década de los años '70 marcó el inicio del proceso de urbanización con un ritmo de crecimiento mucho más acelerado que el del total del territorio nacional. En la tabla № 1 se puede advertir que a mitad de Siglo XX -censo de 1947- cuando el país presentaba un $62,2 \%$ de población urbana, el Chaco sólo registraba $30,1 \%$, mientras que según datos del último censo esta diferencia que en aquel momento fue de más de $32,1 \%$ pasó a ser sólo de 3,2\% en el último recuento nacional. Así se observa claramente que el ritmo de ascenso de la población urbana chaqueña es mayor que el del país.

En contraposición a ello, en el mismo cuadro se advierte la tendencia decreciente de la población rural, por cuanto inicia la serie de datos recabados -censo 1947- con un 69,9\% de población rural y finaliza la serie con un $12,2 \%$, respecto del total de población provincial. La importancia de este fenómeno debe ser visto no solo desde el punto de vista demográfico sino también por las implicancias que trae aparejado en el ámbito urbano, por cuanto provoca variaciones en la demanda de servicios públicos como educación, salud, transporte, agua potable, cuya ausencia y/o deficiencia genera malestar en la población e incluso generar conflictos sociales recurrentes.

\section{Variación de la distribución de la Población Rural, 2001-2010}

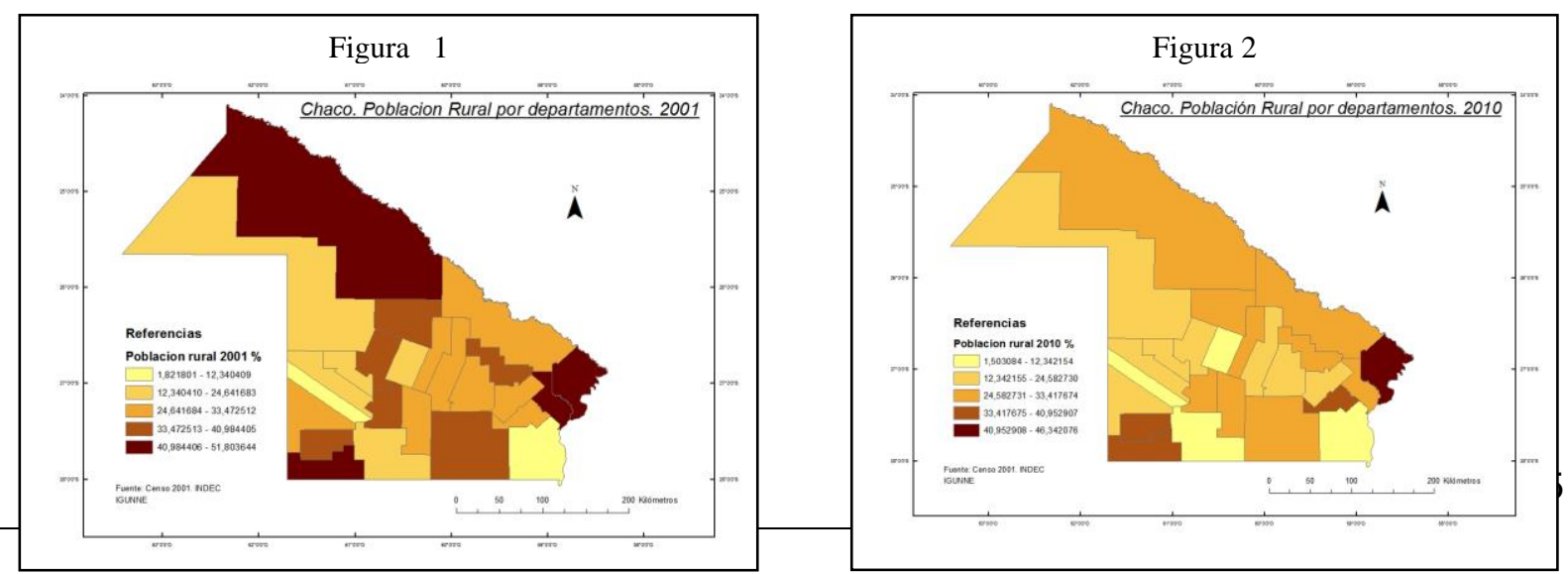

Publicado en formato digital: Prof. Mgtr. Viviana Claudia Pertile. EL DESARROLLO RURAL EN LA PROVINCIA DEL CHACO: FUNDAMENTOS Y VARIABLES DE ANÁLISIS. Revista Geográfica Digital. IGUNNE. Facultad de Humanidades. UNNE. Año 14. № 28. Julio - Diciembre 2017. ISSN 1668-5180 Resistencia, Chaco.

En: http://hum.unne.edu.ar/revistas/geoweb/default.htm 
En las figuras № 1 y 2 mostramos la distribución territorial de la población rural a nivel departamental.

La cartografía resultante revela claramente los sectores de la provincia que más población rural perdieron entre los dos últimos censos nacionales de población, entre los que se destacan los departamentos de 1ํㅡㄹ de Mayo, General Güemes, Independencia, Maipú, O’Higgins, 9 de Julio, General Belgrano, entre los más significativos.

Por su parte la Figura 3 muestra la variación intercensal de la población rural entre los dos últimos censos. Claramente se advierte que los departamentos que mayor proporción de

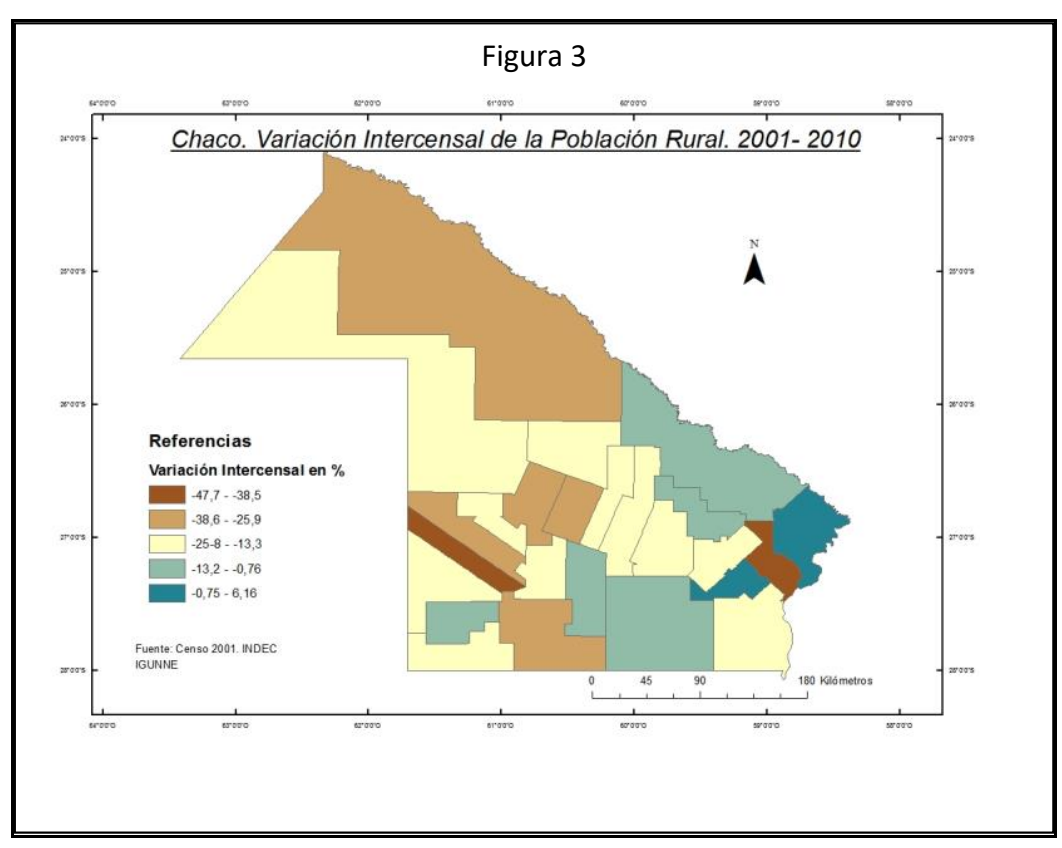
población rural perdieron son los de $1^{\circ}$ de Mayo y Chacabuco con valores entre $47 \mathrm{y}$ $38 \%$, le siguen los departamentos de Comandante Fernández, Independencia, 9 de Julio y Mayor Luis J. Fontana con valores porcentuales negativos entre 38 y $28 \%$.

En las décadas del 70 y 80' éstos departamentos concentraban altas proporciones de población rural, atraídas por el cultivo del algodón, en tanto que en las décadas siguientes, este cultivo fue reemplazado por el cultivo de la soja dejando a una alta proporción de población rural sin trabajo debido a la mecanización que demanda este cultivo.

A partir de la década del $90^{\prime}$ se producen importantes cambios como consecuencia de la ampliación de la frontera agropecuaria entre los que se pueden mencionar:

- Presencia de grandes productores, algunos de los cuales tenían entre mil y cinco mil hectáreas dedicadas a la agricultura, pero también es preciso mencionar la existencia de megaproducciones que alcanzan las 10 mil hectáreas.

- Multiplicación de las desmotadoras privadas en Chaco y Santiago del Estero, cuya capacidad de desmote cubría a principios de los 90'el 83\% del total del país, la contrapartida de este hecho lo constituyó el deterioro económico de las Cooperativas y Pymes.

- La tecnificación agrícola, representada fundamentalmente por la cosecha mecánica, gracias al abaratamiento de las maquinarias nacionales e importadas. En este punto, debemos mencionar consecuencias negativas desde el punto de vista social y económico por cuanto no sólo se han reducido notablemente los desplazamientos de

Publicado en formato digital: Prof. Mgtr. Viviana Claudia Pertile. EL DESARROLLO RURAL EN LA PROVINCIA DEL CHACO: FUNDAMENTOS Y VARIABLES DE ANÁLISIS. Revista Geográfica Digital. IGUNNE. Facultad de Humanidades. UNNE. Año 14. № 28. Julio - Diciembre 2017. ISSN 1668-5180 Resistencia, Chaco. 
los cosecheros, con las implicancias que ello genera en el circuito económico general, sino también que miles de ellos quedaron sin trabajo al desaparecer prácticamente la cosecha manual.

- Desaparición del pequeño productor del circuito productivo, ante la imposibilidad competitiva y de lograr rentabilidad debido a la escasez de tierra para cultivar. Pertile, op cit (2004):

Estos cambios fueron significativos a juzgar por las consecuencias mencionadas. En primer lugar la cuestión referida al modelo de ocupación del territorio, en donde se advirtió una fuerte caída del número los pequeños productores (dedicados al cultivo de algodón) e incremento de las medianas y grandes producciones dedicadas a los cultivos transgénicos como soja, maíz, girasol, entre otros, lo que podemos sintetizar diciendo que se trató de un proceso de concentración de la tierra (3). Otra de las consecuencias es la concentración de la población rural en las áreas periurbanas, en muchos casos coincidentes con los cinturones de pobreza de las ciudades; también debemos hacer mención al alto índice de contaminación ambiental como consecuencias de los cultivos transgénicos cuya producción sólo está garantizada por uso intensivo de agroquímicos altamente nocivos para el medio y para la población.

\section{Indicadores Sociales}

\section{Necesidades Básicas Insatisfechas (NBI) y otros}

Otra de las variables que resultan significativas a la hora de evaluar los alcances y/o beneficios de la aplicación de planes de desarrollo rural en la provincia del Chaco es el la variación de las Necesidades Básicas Insatisfechas (4). Si bien es cierto que los datos con que contamos provienen del Instituto Nacional de Estadísticas y Censos (INDEC), no debemos dejar de mencionar lo cuestionados que fueron los resultados publicados. De todos modos ha sido esta la fuente con que la mayoría de los investigadores trabajaron y en nuestro caso también recurrimos a las mismas.

A los efectos de describir la variación de esta variable en los últimos dos censos, representamos a nivel departamental la proporción porcentual de NBI por departamento (Figura 4 y 5 ).

En el año 2001 el único departamento que tenía entre 18 y $20 \%$ con NBI era el que

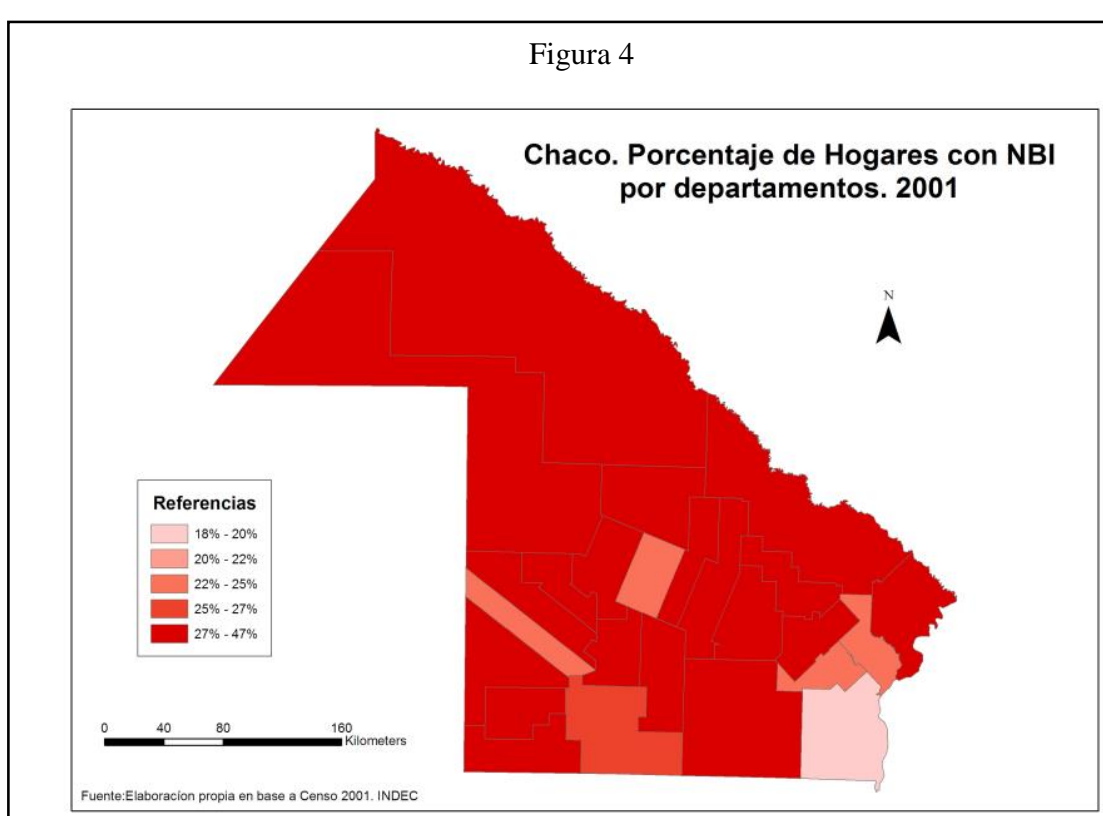
tiene como cabecera a la ciudad capital de la provincia, San Fernando, en tanto que la mayoría de los departamentos registraba proporciones porcentuales que alcanzaban los $47 \%$ 
La figura № 5 representa hogares con NBI en año 2010. Si bien la situación se presentó con valores que nos hablan de una mejora en relación al año 2001, advertimos que más de la

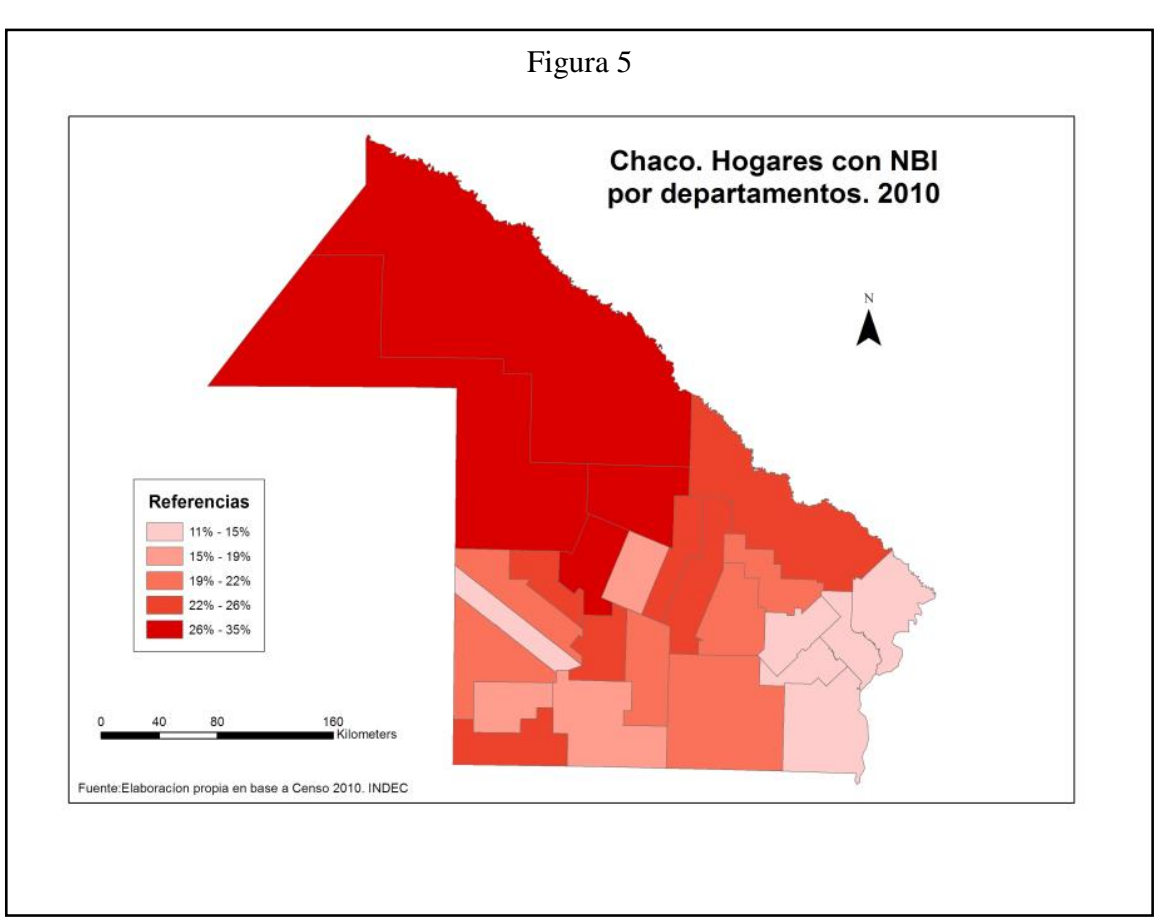
mitad de los presentan valores de NBI superiores al $19 \%$, siendo los que registran la mayor proporción General Güemes, Almirante Brown, Independencia y Maipú.

La situación en el Este provincial, con el departamento San Fernando a la cabeza $(11,4 \%)$ sigue siendo la que presenta la mejor situación, ya que registró los valores con menor proporción de $\mathrm{NBI}$ en el año 2010.

Sintéticamente podríamos decir que, en primer lugar se observa una disminución en los valores porcentuales de las necesidades básicas insatisfechas, fundamentalmente en los departamentos de Este y Centro de la provincia. En segundo lugar, si bien existe una disminución generalizada (Figura 5), no debemos dejar de mencionar la alta proporción de hogares con NBI que se mantienen en los departamentos del Oeste provincial.

Otra de las fuentes consultadas DAR, Desarrollo Argentino, quienes publican datos referidos a esta variable y dan cuenta de la alta proporción a nivel población rural de NBI. Según sus informes el 31,4\% de la población rural de la provincia del Chaco presenta NBI en el año 2010, en tanto que la proporción de población urbana con NBI en la provincia alcanzó para el mismo año un $15.9 \%$.

La Provincia presenta situaciones deficitarias en lo que a vivienda se refiere. Si bien es cierto que dentro de las NBI se tiene en cuenta si se trata de viviendas no apropiadas o hacinamiento, es importante considerar esta variable. Según un informe presentado por Unidad para el Cambio Rural, dependiente del Ministerio de Agricultura, Ganadería y Pesca, se señala que "el parque habitacional resulta precario e insuficiente y sigue,..a esto se agrega la escasa cobertura de los servicios esenciales en las zonas rurales y en las localidades con menor cantidad de habitantes, como así también las dificultades económicas por las que atraviesan las familias para acceder a estos servicios.

Otro de los indicadores mencionados en este mismo informe hace referencia a la situación sanitaria, la que también resulta deficitaria, por cuanto el $65,5 \%$ de la población no posee cobertura de obra social y/o plan de salud mutual o privado, condición vinculada con

Publicado en formato digital: Prof. Mgtr. Viviana Claudia Pertile. EL DESARROLLO RURAL EN LA PROVINCIA DEL CHACO: FUNDAMENTOS Y VARIABLES DE ANÁLISIS. Revista Geográfica Digital. IGUNNE. Facultad de Humanidades. UNNE. Año 14. № 28. Julio - Diciembre 2017. ISSN 1668-5180 Resistencia, Chaco.

En: http://hum.unne.edu.ar/revistas/geoweb/default.htm 
las dificultades de acceso a los servicios de atención de la salud, a la insuficiencia de ingresos y a la informalidad laboral. La tasa de mortalidad infantil es de $27,7 \%$, mientras que la tasa de mortalidad materna es de $8,1 \%$.

\section{Variables económicas y bases productivas}

Analizar las variables económicas y las bases productivas de las áreas rurales en la provincia del Chaco es fundamental por cuanto se constituyen en eje central como generadoras de diversas actividades, empleos e inversiones; como así también demandas de servicios e insumos que impulsan la actividad industrial y comercial en ámbitos rurales locales y por añadidura un crecimiento de la economía en su conjunto.

La estructura económica de nuestra provincia se basó históricamente en la agricultura, la ganadería y la explotación forestal (5), aunque hubo avances de las actividades terciarias.

En los últimos años, según datos proporcionados Secretaría de Planificación y Evaluación de Resultados de la Provincia del Chaco 2010, tabla № 2, el PBG está compuesto fundamentalmente por ramas de Agricultura, Ganadería, Pesca y Silvicultura y las Construcciones, que en conjunto reúnen aproximadamente las dos terceras partes del total del producto provincial. En menor proporción porcentual se encuentra el aporte del sector Transporte, Almacenamiento y Comunicaciones, cuya proporción alcanza el 11,2\% del total, mientras que la Industria Manufacturera y la Administración Pública corresponden cada una aproximadamente a un 5\% del PBG durante el 2010.

\section{Tabla № 2}

\begin{tabular}{|l|l|}
\hline \multicolumn{1}{|c|}{ Ramas de Actividades } & $\%$ \\
\hline Agricultura, ganadería, caza y silvicultura & 32,40 \\
\hline Pesca & 0,003 \\
\hline Explotación de minas y canteras & 0,21 \\
\hline Industrias manufactureras & 4,85 \\
\hline Electricidad, gas y agua & 2,56 \\
\hline Construcciones & 33,0 \\
\hline Comercio & 3,98 \\
\hline Restaurantes y hoteles & 0,59 \\
\hline Transporte, almacenamiento y comunicaciones & 11,18 \\
\hline Servicios financieros & 1,72 \\
\hline Actividades empresariales, inmobiliarias y de alquiler & 2,73 \\
\hline defensa y seguridad social & 5,07 \\
\hline Enseñanza, servicios sociales y de salud & 0,65 \\
\hline Servicios comunitarios, sociales, personales y & 1,06 \\
\hline Total & 100 \\
\hline
\end{tabular}

Fuente: Secretaría de Planificación y Evaluación de Resultados (SPER).

\section{Agricultura}

La estructura agraria chaqueña dependió desde mediados del siglo XX del cultivo algodonero, primacía que perduró hasta principios de los años noventa, momento en el que

Publicado en formato digital: Prof. Mgtr. Viviana Claudia Pertile. EL DESARROLLO RURAL EN LA PROVINCIA DEL CHACO: FUNDAMENTOS Y VARIABLES DE ANÁLISIS. Revista Geográfica Digital. IGUNNE. Facultad de Humanidades. UNNE. Año 14. № 28. Julio - Diciembre 2017. ISSN 1668-5180 Resistencia, Chaco.

En: http://hum.unne.edu.ar/revistas/geoweb/default.htm 
se produjo un proceso de diversificación agrícola como consecuencia de muchos factores, donde además del algodón, se destacaron el girasol, trigo, maíz, sorgo y principalmente soja, quienes se constituyeron en el conjunto de cultivos que predominaron en el espacio rural de la provincia del Chaco.

Pese a la primacía que por décadas tuvo el cultivo del algodón, no debemos dejar de mencionar las crisis por las que atravesó este monocultivo abriendo camino a la incorporación de una nueva propuesta productiva a nivel provincial asociada al cultivo de soja. La inserción del cultivo de soja RR (Roundup Ready) ha sido progresiva y sostenida. El desarrollo de la misma adquirió mayor intensidad en el sudoeste chaqueño, cuyos capitales de inversión fueron y siguen siendo principalmente extraprovinciales, los empresarios foráneos accedieron a las tierras a través de compras o arrendamientos, a muy bajo precio, desplazando al pequeño productor, imposibilitado de acceder a modernas tecnologías que acompañan al cultivo de soja. Pertile y Torre Geraldi (2009)

En el Gráfico № 2 se representa el comportamiento evolutivo de los principales cultivos que comenzaron a desarrollarse a fines de los ochenta y principios de los noventa.

Lo primero que observamos es la destacada participación del cultivo del algodón en la campaña 95/96 en lo que a superficies sembradas se refiere. Esta campaña representó un récord histórico nacional. Los factores que influyeron fueron varios, el más importante fue sin dudas el precio alcanzado por el producto en la campaña anterior, (los índices de precios nacionales fueron más elevados que los internacionales). Otro factor fue la disminución de los costos de insumos y de las maquinarias agrícolas. Estas podían ser adquiridas a valores inferiores a los de la década del 80'. Esto determinó que los productores ampliaran las superficies dedicadas al cultivo.

Es importante destacar que esta campaña no solo representó un récord histórico, sino que también determinó un cambio en los primeros puestos provinciales en lo que concierne a la producción.

A partir de esta campaña, el cultivo del algodón sufre una caída vertiginosa en contraposición de lo que sucede con las superficies sembradas con el cultivo de soja que alcanzaron las 700.000 hectáreas.

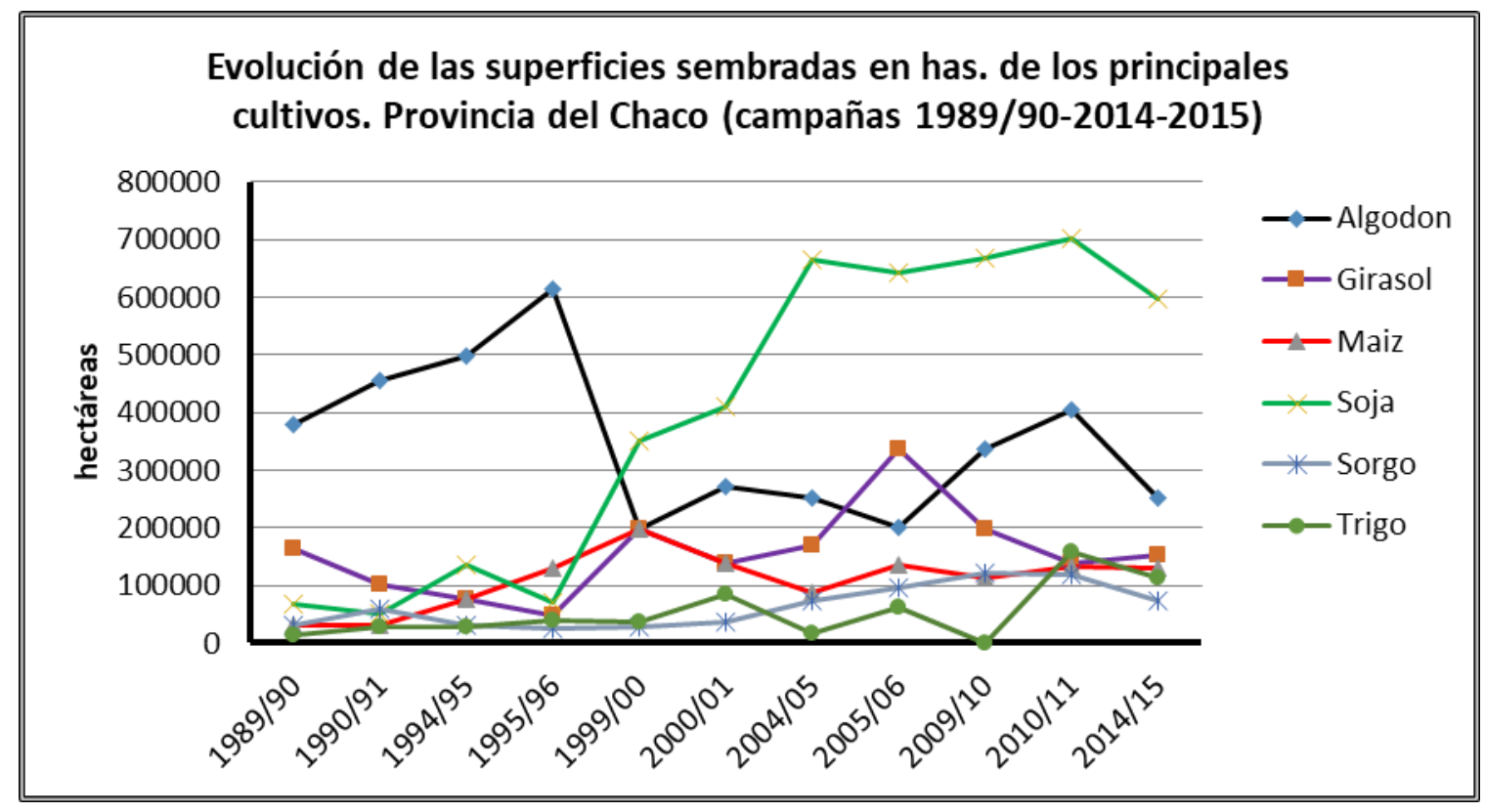

Publicado en formato digital: Prof. Mgtr. Viviana Claudia Pertile. EL DESARROLLO RURAL EN LA PROVINCIA DEL CHACO: FUNDAMENTOS Y VARIABLES DE ANÁLISIS. Revista Geográfica Digital. IGUNNE. Facultad de Humanidades. UNNE. Año 14. № 28. Julio - Diciembre 2017. ISSN 1668-5180 Resistencia, Chaco. 
Revista Geográfica Digital. IGUNNE. Facultad de Humanidades. UNNE. Año 14. № 28.

Julio - Diciembre 2017. ISSN 1668-5180 Resistencia, Chaco

\section{Gráfico № 2: Elaboración propia en base a datos de la SIIA}

Esta situación estuvo acompañada con cambios en la estructura productiva, comienzan a disminuir notablemente el número de pequeños productores y surgen las producciones empresariales dedicadas al cultivo de la soja.

En virtud del análisis realizado con algunas variables tanto demográficas como productivas, cabe preguntarnos ¿cuál es el rol que desempeña/ó el Desarrollo Rural en la Provincia del Chaco?

\section{Para cerrar}

Varios han sido los problemas que, a lo largo de la lectura de los planes y recorridos por diferentes sectores de la provincia del Chaco hemos podido detectar.

En primer lugar debemos decir que, como consecuencia de la diversidad de planes y programas, sean los que han finalizado como los que se encuentran vigentes, determinó la existencia de una superposición de políticas públicas rurales sobre el mismo territorio. Una consecuencia de esta situación es que no solo existe un uso ineficiente de los recursos públicos, sino también una constante dependencia de ayuda estatal y en consecuencia el surgimiento del asistencialismo.

Otra de las problemáticas advertidas es la discontinuidad temporal de las medidas tendientes a resolver problemáticas rurales, convirtiéndose en simples enmiendas en lugar de tratarse de verdaderas acciones tendientes a resolver problemas estructurales.

En cuanto a los problemas que tienen relación con la producción, debemos señalar que los pequeños productores dedicados al cultivo del algodón, siguen enfrentando desde hace unos años escenarios adversos para mejorar su producción -endeudamientos, descapitalización, tecnologías empleadas, entre otras- y por ende sus condiciones de vida.

Muchas veces la dificultad para la diversificación de su producción radica en el desconocimiento o dificultad para acceder a técnicas productivas que le permitan mantenerse en el mercado productivo; este hecho determina que muchos de los pequeños productores continúen

No podemos dejar de mencionar en estas problemáticas, las que involucran a las migraciones campo-ciudad. Es evidente la brecha existente entre los servicios rurales y los urbanos; las diferencias están dadas en la disponibilidad, costo y calidad de los servicios, sean éstos para mejorar la producción o condiciones de vida de los pobladores rurales. En general cuando la población rural emigra a las zonas urbanas, difícilmente regresa al ámbito rural, aún cuando las mejoras sean aparentes, por cuanto la valoración subjetiva ante la posesión de ciertos bienes y servicios se ven contrarrestados por condiciones de vida que suelen empeorar (hacinamiento, marginalidad, deficiente alimentación por insuficiencia de recursos económicos, exposición a la violencia, entre otros.)

La permanente propuesta de alternativas para lograr el desarrollo rural se ha convertido es un instrumento del poder dominante para plantear ilusiones que mantengan a la población rural de menores recursos imaginando un futuro más ventajosos y superador. A través de los años hemos observado que los diferentes gobiernos han garantizado la rentabilidad capitalista asegurando un proceso expansión acelerada de superficies cultivadas y de acumulación y al mismo tiempo una aceptación general de una desigualdad socio-territorial cada vez más marcada, sobre todo de sectores rurales marginales y en particular de la provincia del Chaco, prueba de ello lo constituye el número de población rural cada vez menor habitando el territorio regional y provincial.

En base a la bibliografía y documentación analizada, podemos concluir que tanto los gobiernos de turno como los sectores económicos de poder consideran al desarrollo como 
sinónimo de crecimiento económico en contraposición a la desigualdad social que dicho proceso genera y sin considerar que solamente un cambio radical y estructural puede lograrlo.

Finalmente, a partir de las variables analizadas podemos reforzar las ideas introductorias por cuanto los desplazamientos de las poblaciones rurales dan cuenta de las debilidades de las políticas de desarrollo rural, por cuanto su implementación no sirvió para dar respuesta a las necesidades de una empobrecida población rural.

\section{Referencias}

1.- A partir de diciembre de 2015 hubieron modificaciones en la denominación de las distintas secretarías y subsecretarías. La ex Secretaría de Agricultura Familiar (AF) se fusiona con otras dos secretarías conformándose la Secretaría de Agricultura Familiar, Coordinación y Desarrollo Territorial dividida en tres subsecretarías: Subsecretaría de Agricultura Familiar (AF), Subsecretaría de Coordinación Política y la Subsecretaría de Desarrollo Territorial (DT). El Ministerio de Agricultura, Ganadería y Pesca (MAGyP) de la Argentina es el organismo gubernamental responsable de diseñar y ejecutar planes de producción, comercialización y sanidad en el ámbito agropecuario, pesquero, forestal y agroindustrial.

Hasta 2008, era una Secretaría dependiente del Ministerio de Economía. A partir del 1 de octubre de 2009, fue elevado al rango de Ministerio. Este Ministerio cuenta con la Secretaría de Desarrollo Rural y Agricultura Familiar, de la cual depende la Subsecretaría de Agricultura Familiar y la Subsecretaría de Desarrollo de Economías Regionales.

http://www.minagri.gob.ar/site/institucional/index.php

2.- "La producción de las pequeñas explotaciones se volvieron insuficientes para hacer económicamente rentable la actividad, razón por la cual se vio la necesidad de contar con superficies más grandes para producir en escala, bajar los costos y aumentar la rentabilidad. Esto hizo que la mecanización cosecha se convirtiera en la protagonista de ello, permitiendo la recolección de una producción considerablemente más importante. Durante los años noventa, la mecanización de la recolección ha seguido un ritmo acelerado, casi $90 \%$ de la producción total se recolecta a máquina (pág5) por lo que el polo de desarrollo algodonero se ha desplazado hacia el SO (pág 9)

3.- Según datos del CNA (Censo Nacional Agropecuario) en el intervalo de 1988 y 2002, indican una disminución del $11 \%$ y $32 \%$ de la población rural en los Departamentos de la región Sudoeste de Chaco. Observando los datos censales, en el año 2011 se estima que en las 900.000 hectáreas de la región, se encuentran aproximadamente 1000 pequeñas producciones, con predios de superficies entre media ha hasta50 has. De esta manera observamos un proceso de concentración de la tierra, y de una agricultura sin agricultores. Las pequeñas extensiones de entre 50 a 100 has que en el año 2002 alcanzaban una proporción porcentual de 52\% al decir de Zarrilli, 2008 no pudieron adaptar sus funciones y conocimientos con los requeridos por el nuevo modelo tecnológico productivo; y los productores con menos de 50 hectáreas, que dependieron históricamente de la asistencia del gobierno con subsidios que no permiten el desarrollo del sector, que para cualquier iniciativa de siembra son fuertemente limitados; quedando estos expulsados del modelo de producción y crecimiento económico

4.- Los hogares con Necesidades Básicas Insatisfechas son los hogares que presentan al menos uno de los siguientes indicadores: i) Hacinamiento: más de tres personas por cuarto; ii) Vivienda: habitan en una vivienda de tipo inconveniente (pieza de inquilinato, pieza de hotel o pensión, casilla, local no construido para habitación o vivienda móvil) iii) Condiciones Sanitarias: no tiene ningún tipo de retrete; iv) Asistencia Escolar: tiene al menos un niño en edad escolar ( 6 a 12 años) que no asiste a la escuela; v) Capacidad de Subsistencia: tiene cuatro o más personas por miembro ocupado, cuyo jefe no haya completado el tercer grado de escolaridad primaria.

5.- La ganadería bovina ha sufrido variaciones en el número de cabezas, pero en general en los últimos diez años se mantuvo entre los dos millones y los dos millones seiscientos mil cabezas para el año 2015. La explotación forestal, muy disminuida, continúa con la explotación de todas las especies como así también la del quebracho colorado para mantener dos fábricas de tanino que aún quedan (Puerto Tirol y La Escondida); otra especie muy apreciada es el algarrobo que da lugar a la industria del mueble, asentada principalmente en la localidad de Machagay.

\section{Bibliografía}

Publicado en formato digital: Prof. Mgtr. Viviana Claudia Pertile. EL DESARROLLO RURAL EN LA PROVINCIA DEL CHACO: FUNDAMENTOS Y VARIABLES DE ANÁLISIS. Revista Geográfica Digital. IGUNNE. Facultad de Humanidades. UNNE. Año 14. № 28. Julio - Diciembre 2017. ISSN 1668-5180 Resistencia, Chaco.

En: http://hum.unne.edu.ar/revistas/geoweb/default.htm 
Revista Geográfica Digital. IGUNNE. Facultad de Humanidades. UNNE. Año 14. № 28. Julio - Diciembre 2017. ISSN 1668-5180 Resistencia, Chaco

ARROYO, D. (2012). La pertinaz pobreza (pp. 8-9). Le Monde Diplomatique. Buenos Aires: edición Cono Sur, septiembre.

BAIGORRI, A. (1995): De lo rural a lo urbano, hipótesis sobre las dificultades de mantener la separación epistemológica entre Sociología Rural y Sociología Urbana en el marco del actual Proceso de Urbanización Global. In: V Congreso Español de Sociología. Granada, 1995.

CASTELLS, Manuel (1998). "Entender nuestro mundo", en Revista de Occidente, España. Fundación José Ortega y Gasset.

DAR: Desarrollo Argentino http://www.desarrolloargentino.org/nea/chaco

FERNANDES, Bernardo Mançano (2010). Territorios en disputa: campesinos $y$ agrobusiness. en : http://www.landaction.org/IMG/pdf/Bernardo_halifax_esp.pdf.

INTA 2014: Programa Federal de Apoyo al Desarrollo Rural

http://inta.gob.ar/documentos/profeder-programa-federal

M.A G y Pesca y Unidad para el Cambio Rural, http://www.ucar.gob.ar/index.php/prosap

MANZANAL y SNEIDER (2011) Agricultura Familiar y Políticas de Desarrollo Rural en Argentina y Brasil (análisis comparativo, 1990-2010). Revista Interdisciplinaria de Estudios Agrarios $\mathrm{N}^{\circ} 34$, p. 35-71, CIEA, FCE, UBA, ISSN 1514-1535, 1er semestre 2011, Buenos Aires.

MANZANAL, M. y NARDI, M.A. (2008). "Modelos de intervención de los proyectos de desarrollo rural en Argentina a partir de 1995". En: Schejtman A., y Barsky O. El desarrollo rural en Argentina, un enfoque territorial. Buenos Aires: Siglo XXI. Recuperado de http://www.filo.uba.ar/contenidos/investigacion/institutos/geo/pert/Manzanal\%20Nardi\%20PD R,\%202008.pdf

MANZANAL, Mabel (2011) Desarrollo, territorio y desigualdad en la globalización. Conflictos actuales en la agricultura familiar del nordeste de Misiones, Argentina. En: Revista Mundo Agrario, vol. 12, no 23, segundo semestre de 2011. Universidad Nacional de La Plata. Facultad de Humanidades y Ciencias de la Educación. Centro de Historia Argentina y Americana.

MANZANAL, Mabel (2013). "Poder y desarrollo. Dilemas y desafíos frente a un futuro ¿cada vez más desigual?". En Manzanal, M. y Ponce, M. (2013) La desigualdad ¿del desarrollo?. Controversias y disyuntivas del desarrollo rural en el norte argentino. Ediciones CICCUS, Buenos Aires.

MANZANAL, Mabel y Otros (2011): Poder y conflicto en territorios del Norte Argentino. E: Estudios Socioterritoriales. Revista de Geografía. № 9. ene-jun 2011, pág. 57-81. Universidad Nacional del Centro de la Provincia de Buenos Aires. Facultad de Ciencias Humanas. Centro de Investigaciones Geográficas -CIG.

MANZANAL, Mabel y PONCE, Mariana (2013): La desigualdad ¿del desarrollo?. Controversias y disyuntivas del desarrollo rural en el Norte Argentino. Ediciones CICCUS, Buenos Aires

MINISTERIO DE AGRICULTURA, GANADERÍA Y PESCA. Unidad para el Cambio Rural (2013): Estrategia Provincial para el Sector Agroalimentario. http://www.ucar.gob.ar/index.php/prosap

MINISTERIO DE AGRICULTURA, GANADERÍA Y PESCA: Primer Encuentro Regional de PRODERNEA

En: http://64.76.123.202/new/00/programas/prodernea/publicaciones/publicaciones.php?carpeta =Desarrollo Rural

MINISTERIO DE ECONOMÍA Y PRODUCCIÓN (2003): Los programas de desarrollo rural ejecutados en el ámbito de la SAGPYA. Serie estudios e investigaciones. Buenos Aires 
NEIRA ALVA, Eduardo (1974). "Las políticas de desarrollo regional en América Latina", Planificación regional y urbana en América latina, ILPES-ILDES, Siglo XXI, México. Citado en

PERTILE, V. y TORRE GERALDI, A (2009): "Cambios productivos en el sector agrícola de la provincia del Chaco: la sojización y sus consecuencias socioeconómicas en los pequeños productores rurales a fines del siglo XX". En: MORELLO, Jorge y Andrea F. RODRIGUEZ (editores) El Chaco sin Bosques: La Pampa o el Desierto del futuro GEPAMA- Universidad de Buenos Aires -UNESCO. Editorial Orientación Grafica

PERTILE, Viviana C. (2004) Ampliación de la Frontera agropecuaria Chaqueña: el Oeste Chaqueño y el cultivo algodonero. En: Geográfica Digital № 1 Revista del Instituto de Geografía. Facultad de Humanidades. UNNE (ISSN1668-5180) hum.unne.edu.ar/academica/institutos/geografia/Geo1/default.htm).

PROINDER /DIRECCIÓN DE DESARROLLO AGROPECUARIO (2007): Los pequeños productores en la República Argentina

SCHEJTMAN, Alexander y BERDEGUÉ, Julio (2004). Desarrollo territorial rural. Debates y temas rurales No 1. Rimisp / Centro Latinoamericano para el desarrollo rural, Santiago de Chile. Disponible en http://www.rimisp.org

SCHERJTMAN, Alejandro y BARSKY, Osvaldo (2008): El desarrollo rural en la Argentina. Ed. Siglo XXI. Buenos Aires, Argentina

SECRETARÍA DE AGRICULTURA, GANADERÍA Y PESCA, (1997): Estrategia de Desarrollo Rural Hacia el Siglo XXI. SAGPyA. Buenos Aires.

SOSA VELASQUEZ, Mario (2012): ¿Cómo entender el territorio? Ed. Cara Parens. Universidad Rafael Landívar. Guatemala.

TAMAYO SAENZ, Manuel (1997).El análisis de las políticas Públicas. En: Bañón, Rafael y Carrillo, Ernesto (compiladores). La Nueva Administración Pública, Alianza Universidad, Madrid

ZARRILLI, Adrian (2008) El proceso de agriculturización en las regiones extrapampeanas argentinas: insostenibilidad y límites de un modelo de transformación. La provincia del Chaco (1980-2006) En: XII Congreso de Historia Agraria. Córdoba, 2008 